\title{
Water Use Efficiency Differences in Maize Varieties under Every Furrow and Alternate Furrow Irrigation
}

\author{
Isaac R. Fandika ${ }^{1}$, Grivin Chipula ${ }^{2} \&$ Geoffrey Mwepa $^{3}$ \\ ${ }^{1}$ Kasinthula Agricultural Research Station, Department of Agricultural Research Services, P.O Box 28, \\ Chikwawa, Malawi \\ ${ }^{2}$ Department of Agricultural Engineering, Lilongwe University of Agriculture and Natural Resources, Bunda \\ College Campus, P.O Box 219, Lilongwe, Malawi \\ ${ }^{3}$ Department of Irrigation, P.O Box 30779, Lilongwe, Malawi \\ Correspondence: Isaac R. Fandika, Kasinthula Agricultural Research Station, Department of Agricultural \\ Research Services, P.O Box 28, Chikwawa, Malawi. Tel: 265-999-336-212. E-mail: fandikai@ yahoo.co.uk
}

Received: December 10, 2019 Accepted: January 13, 2020 Online Published: February 7, 2020

doi:10.5539/sar.v9n2p17

URL: https://doi.org/10.5539/sar.v9n2p17

\begin{abstract}
Water-use efficiency (WUE) differences of selected maize varieties under alternate and every furrow irrigation were investigated in a split-plot design trials with three replicates. Alternate furrow (AFI) and Every furrow irrigation (EFI) were main treatments and twenty maize varieties were sub-treatments. Plots were $64 \mathrm{~m}^{2}$ with one maize seed per station spaced at $0.25 \mathrm{~m}$ apart. Crop water use results indicated that EFI consumed more water than the AFI. The AFI reduced crop water consumption by 38 - 45\% compared to EFI. Differences were also prominent in maize varieties' response to AFI. Late maturing maize varieties proved to have minor yield reduction with AFI compared to early and medium maturing maize varieties. WUE $\left(\mathrm{kg} \mathrm{m}^{-3}\right)$ differed with irrigation water application strategy $(\mathrm{P}<0.001)$. AFI had high WUE. A combination of AFI with selection of water efficient maize varieties was a good strategy for improving WUE. The AFI is a promising furrow irrigation water management strategy for water saving. According to farmers experience at five irrigation schemes and on station research, it was concluded that AFI is one of the climate smart irrigation technique that farmer can easily adopt and apply as it saves labour, time water whilst reducing conflict for water among irrigators. It was recommended that AFI be applied fully on early and medium maturing maize varieties within an irrigation interval of 7 days. For late maturing maize varieties, AFI technique should be applied from initial stage to mid stage (up 55 days from planting) then apply EFI at tasselling and silking stages to reduce water stress at this critical stage.
\end{abstract}

Keywords: maize varieties, alternate furrow irrigation, water use efficiency and every furrow irrigation

\section{Introduction}

Irrigating crop fields consumes about $70 \%$ of the freshwater globally (Kayikcioglu, 2012). In developing countries, about $95 \%$ of the total water withdrawal is for agriculture with the demand projected to continue to increase (FAOWATER, 2008). Maize is one of the staple food crops cultivated under irrigation in most developing countries especially in the sub Saharan region. Although maize crop is adapted to a wide range of climatic and edaphic conditions (Sharma and Dass, 2012; Jakhar et al., 2017) and has highest yield potential among cereals (Sharma and Dass, 2012; Kumari et al., 2017), its cultivation during dry season depends on irrigation and residual moisture. During this season, water as one of the main factors of crop production is scarcely sourced from rivers or groundwater. Most irrigation schemes experience physical and economic water scarcity. This water shortage has resulted into increased cost of crop production or loss of crop yield among smallholder farmers globally (FAO, 2007). On the other hand, high energy requirements for lifting irrigation water complicates and aggravates the issue of water scarcity into economic problems at both large and small-scale farm. This cause compelled studies of efficient water strategies to elucidate issues of water scarcity. Studies on deficit irrigation proved high water savings but with trade-offs of reduced yield and quality (Kang, 1999; Stewart, 1993). A well-balanced optimisation of water is supposed to consider regulation of leaf transpiration and root behaviour (Jones et al. 1992; Dass et al., 2016; Kumari et al., 2016). Alternating water application to furrows has proved to regulate the plants' stomata to respond to water deficits in the root-zone 
(Kang et al. 2000; Han and Kang, 2002). Mitchell et al. (1992) defined alternate furrow irrigation (AFI) as an irrigation application strategy where the every - other furrow is shifted by one furrow during every irrigation so that all furrow are irrigated over the course of two irrigations. It can be a viable option for water saving as it allows the water to move laterally and across the bed to the non-irrigated furrow (subbing), reduces the surface area of moist soil, the area of soil evaporation and deep percolation (Singh et al., 2016). Thus, AFI is beneficial for plants' survival and carbon uptake maximisation that later increases both yield and water conservation (Jones, 1980; Cowan et al., 1982). Grain yield maximisation and efficient water use are the major agronomic and economic goals for sustainable irrigation management worldwide. Manageable deficit irrigation technologies that promote both yield and water conservation showed AFI is one of such sustainable irrigation management strategies. However, there no studies that generated information of water use efficiency (WUE) for different maize varieties under AFI. It was hypothesised that a combination of efficient maize variety and irrigation strategy would increasingly save water whilst optimising maize grain yield. Water productivity was reported to increase without reducing grain yield with AFI with less water application (Jones et al., 1992). This paper discussed WUE of selected maize varieties under AFI and every furrow irrigation (EFI) strategies. It is based on the study that evaluated the application of AFI for maize production and productivity in Malawi.

\section{Materials and Methods}

\subsection{Location and Establishment}

The experiment was carried out at both on-Station and on- farm level from 2014 to 2016 winter cropping seasons. The On-station experiment was carried out at Kasinthula Agricultural Research Station in Chikwawa $\left(16^{\circ} 55^{\prime} \mathrm{S}\right.$, $\left.34^{\circ} 50^{\prime} \mathrm{E}\right)$. The on - farm experiment was carried out on seventy On-Farm Demonstrations(OFDs) which were mounted in the five selected irrigation schemes namely - Mpitilira Irrigation Scheme in Salima (1 Mother \& 9 Baby trials), Nanzolo A \& B Irrigation Scheme in Chikwawa (1 Mother \& 9 Baby trials), Chitsukwa Irrigation Scheme in Nsanje (2 Mother \& 18 Baby trials), Bwanje Valley Irrigation Scheme in Dedza (2 Mother \& 18 Baby trials) and Sanambe Irrigation Scheme in Karonga (1 Mother \& 9 Baby trials). The number of farmers participating was equivalent to the number of OFDs implemented from 2015 to 2016.

\subsection{Experimental Design and Irrigation Management}

\subsubsection{On - Station Experiment}

The experiments were laid-out in a split-plot design where two irrigation application strategies - 1) Alternate furrow irrigation (AFI) and Every furrow irrigation (EFI) strategy were main-plot treatments and twenty maize varieties were sub-plot treatments. AFI meant that one of the two neighbouring furrows were alternatively irrigated during consecutive watering or every other furrow irrigation (Sepaskhan \& Ghaseni, 2008). EFI refers to the conventional way of furrow irrigation in Malawi where every furrow is irrigated at every irrigation, basing on the released technologies (Fandika et al., 2007).

EFI received $30-40 \mathrm{~mm}$ irrigation at every irrigation. AFI plots received half of the irrigation in EFI. Irrigation was applied through siphons to furrows and crop water use for irrigated treatments was determined by the soil water balance approach (Allen et.al. 1998), whilst soil moisture measurements were taken by gravimetric methods. The actual water depth within each plot was monitored (at every irrigation) by using a number of siphons. The irrigation depth for a particular plot was determined as an average of the water depth in the siphon from each plot. The amount of water applied to each plot was totalled and recorded for water productivity assessment.

\subsubsection{On - Farm Demonstrations (OFDs)}

The OFDs used a Mother - Baby Approach to participatory re-evaluate seven maize varieties selected from the twenty maize varieties participatory evaluation at Kasinthula Agricultural Research Station. In order to understand the seven-maize response to AFI, farmers assessed them at two irrigation water management strategies,: (1) AFI and (2) EFI. In order to understand AFI performance to nitrogen management, AFI was assessed under different nitrogen management in comparison with other irrigation water management strategies.

\subsubsection{Plot size and Crop Management}

Each plot consisted of 10 rows of $10 \mathrm{~m}$ long spaced at $0.75 \mathrm{~m}$ apart and maize was planted at a spacing of $0.25 \mathrm{~m}$ apart with one seed per planting station for on - station plots. At On-Farm plots were 5 rows of $10 \mathrm{~m}$ long.. Early maturing maize varieties (SC 403, DKC 8033, ZM, 523 PHB 30G79, MRI 514 and DKC 8181), medium maturing maize varieties (SC 537, PAN 53, MH 30, PHB 30G19, DKC 90-53, SC 627, MRI 614 and P2589W) and late maturing crops (SC 719, PAN4M19, MH 31, DKC 90-89, ZM 721 and PAN4M21) were planted for the trials. Fertilizer was applied at $140 \mathrm{kgN} \mathrm{ha}^{-1}$ with $60 \mathrm{kgN} \mathrm{ha}^{-1}$ at planting followed by $80 \mathrm{KgN} \mathrm{ha}^{-1}$ after 21 days 
from planting. The basal dressing fertilizer of 23:21:0 +4S was applied at a uniform rate of $45 \mathrm{KgP} \mathrm{ha}^{-1}$ and 60 $\mathrm{KgN} \mathrm{ha}^{-1}$, respectively. All other crop husbandries were carried out accordingly.

\subsubsection{Crop Water Use and Water Use Efficiency}

A soil water balance method was used to determine the soil moisture deficit (SMD) on a daily basis during the growth of the crops (Premrov et al., 2010). The potential evapotranspiration (ETp) in the soil water balance was determined from evaporation pan (Allen et al., 1998). The crop coefficient factors used in the crop water use computation were for maize as provided by Allen et al. (1998). The daily weather data, for running the soil water balance model, were collected daily from Kasinthula Research Station in Chikwawa, Malawi. The actual crop evapotranspiration $\left(\mathrm{ET}_{\mathrm{c}}\right)$ was determined using equation 1 (Allen et al. 1998). Soil moisture change $(\Delta \mathrm{S})$ was the difference between soil moisture content at the end and the start of the field experiment as measured using gravimetric method. Drainage and surface runoff $\left(R_{0}\right)$ was ignored as the water was under control in the dry season. ETc was referred to as consumption water use (CWU) according to Hoekstra et al. (2009).

$$
\mathrm{ETc}=\mathrm{P}+\mathrm{I}-\mathrm{D}_{\mathrm{p}}-\mathrm{R}_{\mathrm{o}}+\Delta \mathrm{S}
$$

Water Use Efficiency (WUE) was determined as the total grain yield $\left(\mathrm{kg} \mathrm{ha}^{-1}\right)$, per unit of water used $\left(\mathrm{m}^{3} \mathrm{ha}^{-1}\right)$. The economic water productivity index $\left(\mathrm{MK} / \mathrm{m}^{3}\right)$ was assessed as the overall present value of each crop's marketable produce (in MK) divided by the volume of water $\left(\mathrm{m}^{3}\right)$ consumed by the plant (Barker et al., 2003; Molden et al., 2001).

\subsection{Data and Statistical Analysis}

The data on maize grain yield $\left(\mathrm{kg} \mathrm{ha}^{-1}\right)$, water use efficiency and economic water productivity, were analysed with the ANOVA procedure of Genstat statistical package ( $18^{\text {th }}$ Edition) in 2014 to 2016 the differences amongst treatment means were compared with the LSD, at the 5\% probability level (Meier, 2006).

\section{Results \& Discussion}

\subsection{Daily and Cumulative Water Consumption ( $\mathrm{mm}$ ) in Maize}

The winter growing season length for maize in dry season ranged from 110 - 126 days with an average potential water requirement of $555 \mathrm{~mm}, 580 \mathrm{~mm}$ and $521 \mathrm{~mm}$, respectively (Tables $1-2$ ). The mean daily $\mathrm{ET}_{\mathrm{c}}$ for this study period averaged $5.1 \mathrm{~mm} \mathrm{~d}^{-1}$ with a minimum $\mathrm{ET}_{\mathrm{c}}$ of $1.2-2.6 \mathrm{~mm} \mathrm{~d}^{-1}$ and maximum $\mathrm{ET}_{\mathrm{c}}$ of $7.6 \mathrm{~mm} \mathrm{~d}^{-1}$ experienced in the month of July and September, respectively (Fig. 1). The potential water consumption in this study was greater than an average of $423 \mathrm{~mm}$ for winter maize in China where daily $\mathrm{ET}_{\mathrm{c}}$ averaged $3-4 \mathrm{~mm} \mathrm{~d}^{-1}$ between May and June (Kang, 2000). This study indicates that EFI consumed more water $(586-588 \mathrm{~mm})$ than the alternate and fixed furrow irrigation $(317-321 \mathrm{~mm}$ ) (Table $1-8$, Fig. 2). AFI reduced crop water consumption by $38-45 \%$ as reported by Kang et.al. (2000) compared to EFI which was within average potential water consumption. The high daily water use from our study sites are due to high daily $\mathrm{ET}_{\mathrm{c}}$ compared to China, whereas the high actual water use in conversional irrigation is due to its usual goal of meeting the potential water use. The low water use in AFI was also due to their usual goal of optimising water use. The consumption water uses results suggest that where water is scarcer, AFI can be used to save water. 


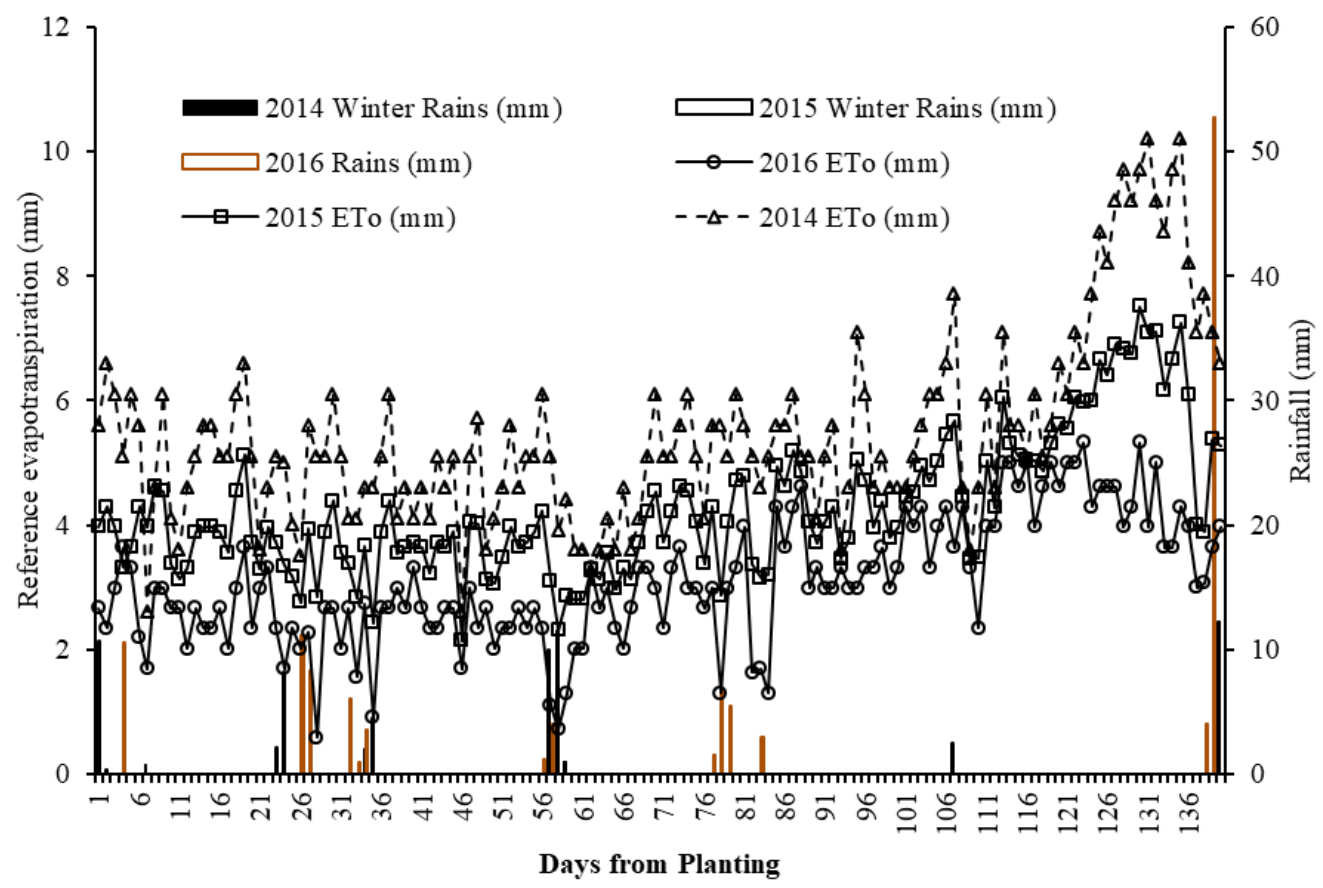

Figure 1. Daily $\mathrm{ET}_{\mathrm{c}}$ and rainfall at Kasinthula Agricultural Research Station during the growing winter season 3.2 Crop Water use for Selected Maize Varieties under AFI and EFI

Maize varieties differ both in terms of their daily water needs and the duration of their total growing period. In this study, consumptive water use $\left(\mathrm{m}^{3} \mathrm{ha}^{-1}\right)$ was greatest in late maturing maize varieties and lowest in early maturing maize varieties', whilst medium maturing maize varieties were intermediate, despite variation within varieties (Table 1a - c). These results on crop water use indicate that maize variety, is also a main factor influencing irrigation water needs. Maize of different maturity differs in their relationship between their maximum water requirement and actual evapo-transpiration, thus crop coefficient $\left(\mathrm{k}_{\mathrm{c}}\right)$ and maturity as their growth stages differ too. Every Furrow irrigation strategy achieved 107\%, 100\% and 94\% of the potential water needs for early, medium and late maturing maize varieties, respectively. AFI strategy was approximately 62, 64 and $63 \%$ of potential water needs for early, medium and late maturing maize varieties, respectively.

Table 1 (a). Crop water use for early maturing maize varieties under AFI and EFI

\begin{tabular}{lllllllll}
\hline Maize Varieties & $\begin{array}{l}\text { Irrigation } \\
(\mathbf{m m})\end{array}$ & $\mathbf{P e}(\mathbf{m m})$ & $\begin{array}{l}\mathbf{D}_{\mathbf{p}} \\
(\mathbf{m m})\end{array}$ & $\begin{array}{l}\mathbf{R}_{\mathbf{o}} \\
(\mathbf{m m})\end{array}$ & $\begin{array}{l}\Delta \mathbf{S} \\
(\mathbf{m m})\end{array}$ & $\begin{array}{l}\text { Potential water } \\
\mathbf{u s e}(\mathbf{m m})\end{array}$ & $\mathbf{E T}_{\mathbf{a}} / \mathbf{E T}_{\mathbf{p}}$ & $\begin{array}{l}\mathbf{C W U} \\
(\mathbf{m m})\end{array}$ \\
\hline Every Furrow Irrigation strategy & & & & & & \\
\hline SC 403 & 374.6 & 37.0 & 0.0 & 0.0 & 36.4 & 448.0 & 1.00 & $\mathbf{4 4 8 . 0}$ \\
DKC 8033 & 372.8 & 35.2 & 0.0 & 0.0 & 6.9 & 414.9 & 1.00 & $\mathbf{4 1 4 . 9}$ \\
ZM 523 & 350.8 & 34.7 & 0.0 & 0.0 & 20.0 & 287.3 & 1.41 & $\mathbf{4 0 5 . 5}$ \\
PHB 30G79 & 375.0 & 37.0 & 0.0 & 0.0 & 56.4 & 468.4 & 1.00 & $\mathbf{4 6 8 . 4}$ \\
MRI 514 & 375.2 & 38.8 & 0.0 & 0.0 & 62.3 & 476.3 & 1.00 & $\mathbf{4 7 6 . 3}$ \\
DKC 8181 & 375.0 & 37.0 & 0.0 & 0.0 & 56.4 & 468.4 & 1.00 & $\mathbf{4 6 8 . 4}$ \\
Mean & & & & & & & $\mathbf{4 . 0 7}$ & $\mathbf{4 4 6 . 9}$ \\
\hline Alternate Furrow Irrigation strategy & & & & & & \\
\hline SC 403 & 187.3 & 37.0 & 0.0 & 0.0 & 36.4 & 448.0 & 0.58 & $\mathbf{2 6 0 . 7}$ \\
DKC 8033 & 186.4 & 35.2 & 0.0 & 0.0 & 6.9 & 414.9 & 0.55 & $\mathbf{2 2 8 . 5}$ \\
ZM 523 & 175.4 & 34.7 & 0.0 & 0.0 & 20.0 & 287.3 & 0.80 & $\mathbf{2 3 0 . 1}$ \\
PHB 30G79 & 187.5 & 37.0 & 0.0 & 0.0 & 56.4 & 468.4 & 0.60 & $\mathbf{2 8 0 . 9}$ \\
MRI 514 & 187.6 & 38.8 & 0.0 & 0.0 & 62.3 & 476.3 & 0.61 & $\mathbf{2 8 8 . 7}$ \\
DKC 8181 & 187.5 & 37.0 & 0.0 & 0.0 & 56.4 & 468.4 & 0.60 & $\mathbf{2 8 0 . 9}$ \\
Mean & & & & & & 0.62 & $\mathbf{2 6 1 . 6}$ \\
\hline
\end{tabular}

Late maturing maize varieties may have high daily water needs apart from having long total growing season that 
made it require more water than early and medium maturing maize varieties which have lowered daily water needs and shorter crop growth seasons. Apart from selecting maize varieties with lower water needs, AFI had proved to reduce the consumptive water in early, medium and late maturity maize varieties by 38,36 and $37 \%$, respectively (Table 1a - c).

Table 1(b). Crop water use for medium maturing maize varieties under AFI and EFI

\begin{tabular}{|c|c|c|c|c|c|c|c|c|}
\hline Maize Variety & $\begin{array}{c}\text { Irrigation } \\
(\mathrm{mm})\end{array}$ & $\mathbf{P e}(\mathbf{m m})$ & Dp & Ro & $\Delta \mathbf{S}$ & $\begin{array}{l}\text { Potential water } \\
\text { use }(\mathrm{mm})\end{array}$ & ETa/ETp & $\begin{array}{l}\text { CWU } \\
(\mathbf{m m})\end{array}$ \\
\hline \multicolumn{9}{|c|}{ Every Furrow Irrigation Strategy } \\
\hline SC 537 & 370.4 & 38.8 & 0.0 & 0.0 & 96.2 & 505.4 & 1.00 & 505.4 \\
\hline PAN 53 & 370.4 & 38.8 & 0.0 & 0.0 & 96.0 & 505.2 & 1.00 & 505.2 \\
\hline MH 30 & 359.9 & 42.6 & 0.0 & 0.0 & 131.4 & 539.4 & 0.99 & 533.9 \\
\hline PHB 30G19 & 419.2 & 46.4 & 0.0 & 0.0 & 84.9 & 550.5 & 1.00 & 550.5 \\
\hline DKC 90-53 & 359.9 & 42.6 & 0.0 & 0.0 & 131.4 & 539.4 & 0.99 & 533.9 \\
\hline SC 627 & 370.2 & 38.8 & 0.0 & 0.0 & 93.5 & 502.5 & 1.00 & 502.5 \\
\hline MRI 614 & 374.1 & 38.8 & 0.0 & 0.0 & 92.2 & 505.1 & 1.00 & 505.1 \\
\hline P2589W & 370.2 & 38.8 & 0.0 & 0.0 & 93.5 & 502.5 & 1.00 & 502.5 \\
\hline Mean & & & & & & & 1.00 & 517.4 \\
\hline \multicolumn{9}{|c|}{ Alternate Furrow Irrigation Strategy } \\
\hline SC 537 & 185.2 & 38.8 & 0.0 & 0.0 & 96.2 & 505.4 & 0.63 & 320.2 \\
\hline PAN 53 & 185.2 & 38.8 & 0.0 & 0.0 & 96.0 & 505.2 & 0.63 & 320.0 \\
\hline MH 30 & 180.0 & 42.6 & 0.0 & 0.0 & 131.4 & 539.4 & 0.66 & 354.0 \\
\hline PHB 30G19 & 209.6 & 46.4 & 0.0 & 0.0 & 84.9 & 550.5 & 0.62 & 340.9 \\
\hline DKC 90-53 & 180.0 & 42.6 & 0.0 & 0.0 & 131.4 & 539.4 & 0.66 & 354.0 \\
\hline SC 627 & 185.1 & 38.8 & 0.0 & 0.0 & 93.5 & 502.5 & 0.63 & 317.4 \\
\hline MRI 614 & 187.1 & 38.8 & 0.0 & 0.0 & 92.2 & 505.1 & 0.63 & 318.1 \\
\hline P2589W & 185.1 & 38.8 & 0.0 & 0.0 & 93.5 & 502.5 & 0.63 & 317.4 \\
\hline Mean & & & & & & & 0.64 & 330.2 \\
\hline
\end{tabular}

Table 1 (c). Crop water use for late maturing maize varieties under AFI and EFI

\begin{tabular}{lllllllll}
\hline $\begin{array}{l}\text { Maize } \\
\text { Variety }\end{array}$ & Irrigation $(\mathbf{m m})$ & $\begin{array}{l}\text { Pe } \\
(\mathbf{m m})\end{array}$ & $\begin{array}{l}\text { Dp } \\
(\mathbf{m m})\end{array}$ & $\begin{array}{l}\text { Ro } \\
(\mathbf{m m})\end{array}$ & $\begin{array}{l}\Delta \mathbf{S} \\
(\mathbf{m m})\end{array}$ & $\begin{array}{l}\text { Potential water } \\
\text { used }(\mathbf{m m})\end{array}$ & ETa/ETp & $\begin{array}{l}\text { CWU } \\
(\mathbf{m m})\end{array}$ \\
\hline \multicolumn{2}{l}{ Every Furrow Irrigation Strategy } & & & & & & & \\
\hline SC 719 & 349.1 & 57.3 & 0.0 & 0.0 & 154.0 & 629.5 & 0.89 & $\mathbf{5 6 0 . 4}$ \\
PAN4M19 & 354.9 & 51.9 & 0.0 & 0.0 & 149.2 & 604.4 & 0.92 & $\mathbf{5 5 6 . 0}$ \\
MH 31 & 354.3 & 46.4 & 0.0 & 0.0 & 145.2 & 569.7 & 0.96 & $\mathbf{5 4 5 . 9}$ \\
DKC 90-89 & 359.9 & 46.4 & 0.0 & 0.0 & 133.5 & 550.1 & 0.98 & $\mathbf{5 3 9 . 8}$ \\
ZM 721 & 351.0 & 51.9 & 0.0 & 0.0 & 152.6 & 610.5 & 0.91 & $\mathbf{5 5 5 . 5}$ \\
PAN4M21 & 352.3 & 46.4 & 0.0 & 0.0 & 147.9 & 573.8 & 0.95 & $\mathbf{5 4 6 . 6}$ \\
Mean & & & & & & & 0.94 & $\mathbf{5 5 0 . 7}$ \\
\hline Alternate Furrow Irrigation Strategy & & & & & & \\
\hline SC 719 & 174.6 & 57.3 & 0.0 & 0.0 & 154.0 & 629.5 & 0.61 & $\mathbf{3 8 5 . 9}$ \\
PAN4M19 & 177.5 & 51.9 & 0.0 & 0.0 & 149.2 & 604.4 & 0.63 & $\mathbf{3 7 8 . 6}$ \\
MH 31 & 177.2 & 46.4 & 0.0 & 0.0 & 145.2 & 569.7 & 0.65 & $\mathbf{3 6 8 . 8}$ \\
DKC 90-89 & 180.0 & 46.4 & 0.0 & 0.0 & 133.5 & 550.1 & 0.65 & $\mathbf{3 5 9 . 9}$ \\
ZM 721 & 175.5 & 51.9 & 0.0 & 0.0 & 152.6 & 610.5 & 0.62 & $\mathbf{3 8 0 . 0}$ \\
PAN4M21 & 176.2 & 46.4 & 0.0 & 0.0 & 147.9 & 573.8 & 0.65 & $\mathbf{3 7 0 . 5}$ \\
Mean & & & & & & 0.63 & $\mathbf{3 7 3 . 9}$ \\
\hline
\end{tabular}

\subsection{Maize Grain Yield and Water Use Efficiency for Selected Maize Varieties at On-Station}

In 2014, crop water use averaged 1,733 $\mathrm{m}^{3} \mathrm{ha}^{-1}$ and 4,230 $\mathrm{m}^{3} \mathrm{ha}^{-1}$ under AFI and EFI, respectively (Table 1 and Table 6). The average maize water uses in 2015 - 2016 was categorised according to maize variety duration short duration varieties used 4,394 $\mathrm{m}^{3} \mathrm{ha}^{-1}$ and 4,469 $\mathrm{m}^{3} \mathrm{ha}^{-1}$ under EFI and 2,495 $\mathrm{m}^{3} \mathrm{ha}^{-1}$ and 2,616 $\mathrm{m}^{3} \mathrm{ha}^{-1}$ under AFI strategy, respectively. The medium maturing maize varieties used $5,439 \mathrm{~m}^{3} \mathrm{ha}^{-1}$ and $5,174 \mathrm{~m}^{3} \mathrm{ha}^{-1}$ of water 
under EFI whilst AFI used 3,327 $\mathrm{m}^{3} \mathrm{ha}^{-1}$ and 3,302 $\mathrm{m}^{3} \mathrm{ha}^{-1}$ in 2015 and 2016 , respectively. The late maturing maize varieties used $6,087 \mathrm{~m}^{3} \mathrm{ha}^{-1}$ and $5,507 \mathrm{~m}^{3} \mathrm{ha}^{-1}$ of water under EFI whilst AFI used 4,024 $\mathrm{m}^{3} \mathrm{ha}^{-1}$ and 3,739 $\mathrm{m}^{3} \mathrm{ha}^{-1}$ in 2015 and 2016, respectively. The AFI reduced water use by 59\% in 2014, $41-43 \%$ among short duration maize varieties, 36 - 54\% among medium duration maize varieties and $32-34 \%$ among late duration maize varieties in 2015 and 2016, respectively. This reduction in water use indicates significant water saving by using AFI strategy.

Table 2. Effect of alternate furrow irrigation on different maize varieties grain yield and water use efficiency at Kasinthula Agricultural Research Station, 2014

\begin{tabular}{|c|c|c|c|c|c|c|c|c|}
\hline \multirow{2}{*}{$\begin{array}{l}\text { Irrigation/ } \\
\text { Varieities }\end{array}$} & \multicolumn{4}{|c|}{ Full Irrigation } & \multicolumn{4}{|c|}{ Alternate Furrow irrigation } \\
\hline & $\begin{array}{l}\text { Irrigation } \\
\left(\mathrm{m}^{3} \mathrm{ha}^{-1}\right)\end{array}$ & $\begin{array}{l}\text { Grain } \\
\text { yield } \\
\left(\mathrm{kgha}^{-1}\right)\end{array}$ & $\begin{array}{l}\text { WUE } \\
\left(\mathrm{kgm}^{-3}\right)\end{array}$ & $\begin{array}{l}\text { NUE } \\
\left(\mathrm{kg}^{-1}\right) \\
\left.\mathrm{kgN}^{-1}\right)\end{array}$ & $\begin{array}{l}\text { Irrigation } \\
\left(\mathrm{m}^{3} \mathrm{ha}^{-1}\right)\end{array}$ & $\begin{array}{l}\text { Grain yield } \\
\left(\mathrm{kg} \mathrm{ha}^{-1}\right)\end{array}$ & $\begin{array}{l}\text { WUE } \\
\left(\mathrm{kgm}^{-3}\right)\end{array}$ & $\begin{array}{l}\text { NUE } \\
\left(\mathrm{kg}^{-1}\right) \\
\left.\mathrm{kgN}^{-1}\right)\end{array}$ \\
\hline Pan 53 & 1732.7 & 5190 & 3.39 & 51.9 & 867.13 & 4306 & 4.45 & 43.1 \\
\hline DKC 9089 & 1732.7 & 4319 & 2.50 & 38.8 & 867.13 & 3881 & 4.47 & 43.2 \\
\hline DKC8053 & 1732.7 & 4827 & 2.79 & 48.2 & 867.13 & 3652 & 4.21 & 38.5 \\
\hline SC627 & 1732.7 & 5873 & 3.40 & 57.0 & 867.13 & 5309 & 6.12 & 54.8 \\
\hline ZM523 & 1732.7 & 4309 & 2.50 & 40.3 & 867.13 & 3525 & 4.07 & 38.1 \\
\hline SC403 & 1732.7 & 3838 & 2.22 & 38.1 & 867.13 & 3606 & 4.16 & 36.4 \\
\hline SC719 & 1732.7 & 5536 & 3.19 & 55.3 & 867.13 & 4678 & 5.40 & 46.8 \\
\hline DK8033 & 1732.7 & 4503 & 2.60 & 45.6 & 867.13 & 3680 & 4.24 & 36.8 \\
\hline DKC9053 & 1732.7 & 5035 & 2.91 & 50.3 & 867.13 & 4184 & 4.83 & 41.8 \\
\hline PHB30G19-6 & 1732.7 & 6063 & 3.50 & 60.6 & 867.13 & 5480 & 6.32 & 54.8 \\
\hline $\operatorname{Mean}(\mathbf{n}=)$ & 1732.7 & 4949 & 2.90 & 48.6 & 867.13 & 4230 & 4.83 & 43.4 \\
\hline $\mathrm{CV}(\%)$ & & & & & & 9.7 & 10.9 & \\
\hline \multicolumn{9}{|l|}{ Significance } \\
\hline Variety & & & & & & $\mathrm{P}<0.001$ & $\mathrm{P}<0.001$ & $\mathrm{P}<0.001$ \\
\hline Irrigation & & & & & & $\mathrm{P}<0.05$ & $\mathrm{P}<0.01$ & NS \\
\hline Interactions & & & & & & Ns & $\mathrm{P}<0.05$ & Ns \\
\hline \multirow[t]{3}{*}{$\mathrm{LSD}_{0.05}$} & Variety & & & & & 518.8 & 0.4919 & \\
\hline & Irrigation & & & & & 515.4 & 0.6390 & - \\
\hline & Interactions & & & & & - & 0.7310 & - \\
\hline
\end{tabular}

Table 2 indicates that there were significant differences between EFI and AFI irrigated maize varieties on average grain yield $(\mathrm{P}<0.001)$ and water use efficiency $(\mathrm{P}<0.001)$. However, there was no significant differences on NUE between EFI and AFI irrigated maize varieties. The maize varieties under EFI had increased grain yield by $16.9 \%$. Nevertheless, WUE increased with AFI strategy. Maize varieties strongly influenced grain yield $(\mathrm{P}<0.001)$, WUE $(\mathrm{P}<0.001)$ and NUE $(\mathrm{P}<0.001)$. The highest grain yield, WUE and NUE was found in PHB30G19-6 whilst SC403 had the least grain yield in 2014. The Drought Intensity Index (DII) was 0.15, thus more than 0.07 cut - off point for water stress. The percentage of yield reduction with water stress caused by AFI strategy in 2014 averaged 14\%. The yield reduction with water stress was highest in DKC8053 and least in DKC9089 as show in fig. 2.

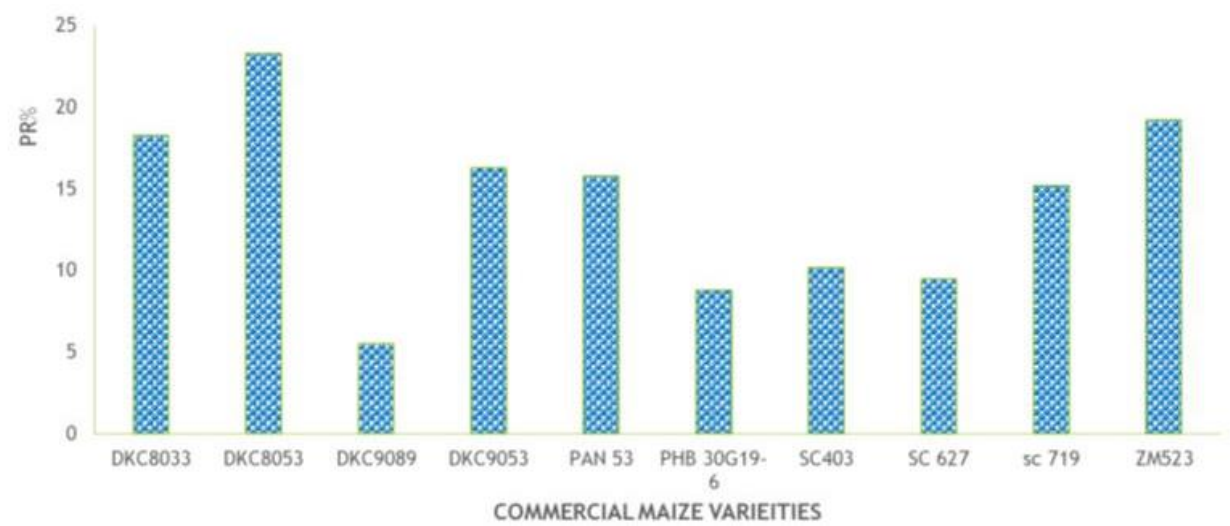

Figure 2. Percentage of yield reduction with water stress in commercial maize varieties 
Table 3 - 5 pointed out that there were no significant differences between EFI and AFI irrigated short and medium duration maize varieties on average grain yield $(\mathrm{P}>0.001)$, on the other hand, the EFI and AFI strategies significantly differed in grain yields among the late maturing maize varieties in $2015(\mathrm{P}<0.001)$. The late maturing maize varieties under EFI had higher grain yields $\left(7,774 \mathrm{~kg} \mathrm{ha}^{-1}\right)$ than those under AFI $\left(6,264 \mathrm{~kg} \mathrm{ha}^{-1}\right)$. Early maturing maize varieties did not differ in grain yields $(\mathrm{P}>0.05)$, however, medium and late maturing maize varieties differed among themselves in response to irrigation strategies in 2015. PAN 53 had highest grain yields under both $\operatorname{EFI}\left(8,155 \mathrm{kgha}^{-1}\right)$ and AFI strategies $\left(7,382 \mathrm{kgha}^{-1}\right)$ among medium maturing maize varieties but not significantly different to DKC $9053\left(7,138 \mathrm{~kg} \mathrm{ha}^{-1}\right)$ under EFI and DKC 8053 under AFI $\left(6,004 \mathrm{~kg} \mathrm{ha}^{-1}\right)$.

The 2015 results suggested that AFI strategy is more suitable to early and medium maturing maize varieties but not to late maturing varieties. The possible reason for this is that late maturing varieties required more water than early and medium maturing maize varieties as a result their response to water stress was greater than the other two groups. However, the 2015 findings differed with 2016 results as significant difference between both irrigation strategies and maize varieties among the medium and late maturing categories $(\mathrm{P}<0.001)$ were determinedbut not among early maturing varieties $(\mathrm{P}>0.05)$. The likely cause for this difference might be weather variability that might contribute to differences in crop water use. The 2016 results also showed that maize grain yields were higher under EFI than under AFI strategies.

Table 3. Grain yield, Water Use Efficiency and Economic Water Productivity for selected early maturing maize varieties under full and alternate furrow irrigation in Malawi

\begin{tabular}{|c|c|c|c|c|c|c|c|c|}
\hline \multirow{2}{*}{$\begin{array}{l}\text { Irrigation/ } \\
\text { Maize } \\
\text { Variety }\end{array}$} & \multicolumn{4}{|c|}{ Winter 2015} & \multicolumn{4}{|c|}{ Winter 2016} \\
\hline & $\begin{array}{l}\text { CWU } \\
\left(\mathbf{m}^{3} \mathbf{h a}^{-1}\right)\end{array}$ & $\begin{array}{l}\text { Grain } \\
\text { yield } \\
\left(\text { Kgha }^{-1}\right) \\
\end{array}$ & $\begin{array}{l}\text { WUE } \\
\left(\mathbf{k g}^{-1}\right) \\
\left.\mathbf{m m}^{-1}\right)\end{array}$ & $\begin{array}{l}\text { EWP } \\
\left(\mathbf{M K} / \mathbf{m}^{3}\right)\end{array}$ & $\begin{array}{l}\text { CWU } \\
\left(\mathbf{m}^{3} \mathbf{h a}^{-1}\right)\end{array}$ & $\begin{array}{l}\text { Grain } \\
\text { yield } \\
\left(\text { Kgha }^{-1}\right)\end{array}$ & $\begin{array}{l}\text { WUE } \\
\left(\mathrm{kgmm}^{-1}\right)\end{array}$ & $\begin{array}{l}\text { EWP } \\
\left(\mathbf{M K} / \mathbf{m}^{3}\right)\end{array}$ \\
\hline \multicolumn{9}{|c|}{ Every Furrow Irrigation } \\
\hline SC 537 & 4,644 & 7,606 & 16.4 & 328 & 4,480 & - & - & - \\
\hline DKC 8033 & 4,307 & 7,281 & 16.9 & 338 & 4,149 & 7,417 & $17.9^{c}$ & $303.89^{c}$ \\
\hline ZM 523 & 3,928 & 7,453 & 19.0 & 380 & 4,055 & 9,083 & $22.4^{\mathrm{bc}}$ & $380.81^{b c}$ \\
\hline PHB 30G79 & - & - & - & - & 4,684 & - & - & - \\
\hline MRI 514 & - & - & - & - & 4,763 & 7,271 & $15.3^{\mathrm{d}}$ & $259.51^{\mathrm{d}}$ \\
\hline DKC 8181 & - & - & - & - & 4,684 & 9,031 & $19.3^{\mathrm{c}}$ & $327.78^{\mathrm{c}}$ \\
\hline MH 18 & 4,695 & 7,009 & 14.9 & 299 & - & - & - & - \\
\hline Mean & 4,394 & 7,336 & 16.8 & 336 & 4,469 & $8,201^{\mathrm{A}}$ & 18.7 & 318.00 \\
\hline \multicolumn{9}{|c|}{ Alternate Furrow Irrigation } \\
\hline SC 537 & 2,759 & 5,620 & 20.4 & 407 & 2,607 & - & - & - \\
\hline DKC 8033 & 2,353 & 6,094 & 25.9 & 518 & 2,285 & 6,251 & $27.4^{\mathrm{a}}$ & $464.99^{\mathrm{a}}$ \\
\hline ZM 523 & 2,182 & 5,287 & 24.2 & 485 & 2,301 & 6,344 & $27.6^{\mathrm{a}}$ & $468.68^{\mathrm{a}}$ \\
\hline PHB 30G79 & - & - & - & - & 2,809 & - & - & - \\
\hline MRI 514 & - & - & - & - & 2,887 & 6,881 & $23.8^{\mathrm{ab}}$ & $405.20^{\mathrm{ab}}$ \\
\hline DKC 8181 & - & - & - & - & 2,809 & 7,490 & $26.7^{\mathrm{a}}$ & $453.27^{\mathrm{a}}$ \\
\hline MH 18 & 2,686 & 5,518 & 20.5 & 411 & - & - & - & - \\
\hline Mean & 2,495 & 5,629 & 22.8 & 455 & 2,616 & $6,741^{B}$ & 26.4 & 448.03 \\
\hline Cv. (\%) & & 17.2 & 19.7 & 19.7 & & 15.6 & 15.1 & 15.1 \\
\hline \multicolumn{9}{|l|}{ Significance } \\
\hline Varieties & & Ns & Ns & Ns & & Ns & $\mathrm{p}<0.05$ & $\mathrm{p}<0.05$ \\
\hline Irrigation & & Ns & Ns & Ns & & $\mathrm{p}<0.05$ & $\mathrm{p}<0.01$ & $\mathrm{p}<0.01$ \\
\hline \multicolumn{9}{|l|}{$\mathbf{L S D}_{0.05}$} \\
\hline Varieties & & - & - & - & & - & 3.58 & 60.78 \\
\hline Irrigation & & - & - & - & & 1395.4 & 3.65 & 62.09 \\
\hline
\end{tabular}

Note: Columns with similar letters are not significantly different.

Rows with similar capital letters are not significantly different.

Water use efficiency and Economic water productivity (EWP) were significantly different between maize under AFI and EFI in both 2015 and 2016 ( $\mathrm{P}<0.001)$ except among short duration maize varieties where irrigation and varieties had no significant impact on WUE and EWP in 2015 ( $\mathrm{P}>0.05$; Table 3 - 5). Despite increased grain yield by $16.9 \%$ with EFI, WUE increased with AFI strategy. Overall, on station results suggested that AFI 
strategy does save water without significantly affecting maize grain yield among early and medium maturing maize varieties but with significant effect on late maturing maize grain yields.

Table 4. Grain yield, Water Use Efficiency and Economic Water Productivity for selected medium maturing maize varieties under full and alternate furrow irrigation in Malawi

\begin{tabular}{|c|c|c|c|c|c|c|c|c|}
\hline \multirow{2}{*}{$\begin{array}{l}\text { Irrigation/ } \\
\text { Maize } \\
\text { Variety }\end{array}$} & \multicolumn{4}{|c|}{ Winter 2015} & \multicolumn{4}{|c|}{ Winter 2016} \\
\hline & $\begin{array}{l}\text { CWU } \\
\left(\mathbf{m}^{3} \mathbf{h a}^{-1}\right)\end{array}$ & $\begin{array}{l}\text { Grain } \\
\text { Yield } \\
\left(\text { Kgha }^{-1}\right)\end{array}$ & $\begin{array}{l}\text { WUE } \\
\left(\mathbf{k g}^{-1}\right) \\
\left.\mathbf{m m}^{-1}\right)\end{array}$ & $\begin{array}{l}\text { EWP } \\
\left(\mathrm{MK} / \mathbf{m}^{3}\right)\end{array}$ & $\begin{array}{l}\text { CWU } \\
\left(\mathbf{m}^{3} \mathbf{h a}^{-1}\right)\end{array}$ & $\begin{array}{l}\text { Grain } \\
\text { Yield } \\
\left(\mathrm{Kg} \mathrm{ha}^{-1}\right)\end{array}$ & $\begin{array}{l}\text { WUE } \\
(\mathbf{k g} \\
\left.\mathbf{m m}^{-1}\right)\end{array}$ & $\begin{array}{l}\text { EWP } \\
\left(\mathbf{M K} / \mathbf{m}^{3}\right)\end{array}$ \\
\hline \multicolumn{9}{|c|}{ Full Irrigation } \\
\hline SC 537 & 5,239 & $6,731^{\mathrm{bc}}$ & $12.9^{\mathrm{c}}$ & $257^{\mathrm{c}}$ & 5,054 & $7,906^{\mathrm{bc}}$ & $15.6^{\mathrm{de}}$ & $265.94^{\mathrm{de}}$ \\
\hline PAN 53 & 5,207 & $8,155^{\mathrm{a}}$ & $15.7^{\mathrm{b}}$ & $313^{\mathrm{b}}$ & 5,052 & $9,385^{\mathrm{a}}$ & $18.6^{\mathrm{cd}}$ & $315.82^{\text {cd }}$ \\
\hline MH 30 & - & - & - & - & 5,339 & $7,708^{\mathrm{bc}}$ & $14.4^{\text {de }}$ & $245.44^{\text {de }}$ \\
\hline PHB 30G19 & - & - & - & - & 5,505 & $8,708^{\mathrm{ab}}$ & $15.8^{\mathrm{de}}$ & $268.92^{\mathrm{de}}$ \\
\hline DKC 90-53 & 5,584 & $7,138^{\mathrm{ab}}$ & $12.8^{\mathrm{c}}$ & $256^{\mathrm{c}}$ & 5,339 & $7,208^{\mathrm{c}}$ & $13.5^{\mathrm{e}}$ & $229.52^{\mathrm{e}}$ \\
\hline DKC 8053 & 5,584 & $6,650^{\mathrm{bc}}$ & $11.9^{\mathrm{c}}$ & $238^{\mathrm{c}}$ & - & - & - & - \\
\hline SC 627 & - & - & - & - & 5,025 & $8,521^{\mathrm{ab}}$ & $17.0^{\mathrm{cd}}$ & $288.27^{\mathrm{cd}}$ \\
\hline MRI 614 & - & - & - & - & 5,051 & $7,719^{\mathrm{bc}}$ & $15.3^{\mathrm{de}}$ & $259.79^{\mathrm{de}}$ \\
\hline P2589W & - & - & - & - & 5,025 & $9,380^{\mathrm{a}}$ & $18.7^{\text {cd }}$ & $317.52^{\mathrm{cd}}$ \\
\hline ZM 623 & 5,580 & $6,412^{\mathrm{bc}}$ & $11.5^{\mathrm{c}}$ & $230^{\mathrm{c}}$ & - & - & - & - \\
\hline Mean & 5,439 & 7,018 & $12.9^{B}$ & $259^{B}$ & 5,174 & 8,318 & 16.1 & 273.90 \\
\hline \multicolumn{9}{|c|}{ Alternate Furrow Irrigation } \\
\hline SC 537 & 3,129 & $5,191^{\mathrm{c}}$ & $16.6^{\mathrm{b}}$ & $332^{\mathrm{b}}$ & 3,202 & $7,583^{\mathrm{bc}}$ & $23.7^{\mathrm{a}}$ & $402.61^{\mathrm{a}}$ \\
\hline PAN 53 & 3,107 & $7,382^{\mathrm{ab}}$ & $23.8^{\mathrm{a}}$ & $475^{\mathrm{a}}$ & 3,200 & $7,427^{\mathrm{bc}}$ & $23.2^{\mathrm{ab}}$ & $394.56^{\mathrm{ab}}$ \\
\hline MH 30 & - & - & - & - & 3,540 & $6,688^{\text {cd }}$ & $18.9^{\text {cd }}$ & $321.15^{\mathrm{cd}}$ \\
\hline PHB 30G19 & - & - & - & - & 3,409 & $6,833^{\mathrm{c}}$ & $20.0^{\mathrm{b}}$ & $340.76^{\mathrm{b}}$ \\
\hline DKC 90-53 & 3,474 & $5,799^{\mathrm{c}}$ & $16.7^{\mathrm{b}}$ & $334^{\mathrm{b}}$ & 3,540 & $5,510^{\mathrm{e}}$ & $15.6^{\mathrm{de}}$ & $264.62^{\text {de }}$ \\
\hline DKC 8053 & 3,474 & $6,004^{\mathrm{bc}}$ & $17.3^{\mathrm{b}}$ & $346^{\mathrm{b}}$ & - & - & - & - \\
\hline SC 627 & - & - & - & - & 3,174 & $6,583^{\text {cd }}$ & $20.7^{\mathrm{bc}}$ & $352.6^{\mathrm{bc}}$ \\
\hline MRI 614 & - & - & - & - & 3,181 & $6,427^{\mathrm{c}}$ & $20.2^{\mathrm{bc}}$ & $343.48^{\mathrm{bc}}$ \\
\hline P2589W & - & - & - & - & 3,174 & $7,250^{\mathrm{c}}$ & $22.8^{\mathrm{ab}}$ & $388.31^{\mathrm{ab}}$ \\
\hline ZM 653 & 3,462 & 5,877 & $17.0^{\mathrm{b}}$ & $340^{\mathrm{b}}$ & - & - & - & - \\
\hline Mean & 3,329 & 6,050 & $18.3^{A}$ & $365^{A}$ & 3,302 & 6,788 & 20.6 & 351.01 \\
\hline Cv. (\%) & & 16.1 & 15.8 & 15.8 & & 16.8 & 17.3 & 17.3 \\
\hline \multicolumn{9}{|l|}{ Significance } \\
\hline Varieties & & $\mathrm{P}<0.05$ & $\mathrm{P}<0.01$ & $\mathrm{P}<0.001$ & & $\mathrm{P}<0.05$ & $\mathrm{P}<0.01$ & $\mathrm{P}<0.01$ \\
\hline Irrigation & & Ns & $\mathrm{P}<0.05$ & $\mathrm{P}<0.05$ & & $\mathrm{P}<0.01$ & $\mathrm{P}<0.001$ & $\mathrm{P}<0.001$ \\
\hline \multicolumn{9}{|l|}{$\mathbf{L S D}_{0.05}$} \\
\hline Varieties & & $1,086.9$ & 2.55 & 51.0 & & $1,277.14$ & 3.22 & 54.69 \\
\hline Irrigation & & - & 3.81 & 76.1 & & 480.73 & 1.16 & 19.77 \\
\hline
\end{tabular}

Note: Columns with similar letters are not significantly different.

Rows with similar capital letters are not significantly different. 
Table 5. Grain yield, water use efficiency and economic water productivity for selected late maturing maize varieties under full and alternate furrow irrigation in Malawi

\begin{tabular}{|c|c|c|c|c|c|c|c|c|}
\hline \multirow{2}{*}{$\begin{array}{l}\text { Irrigation/ } \\
\text { Maize } \\
\text { Variety }\end{array}$} & \multicolumn{4}{|c|}{ Winter 2015} & \multicolumn{4}{|c|}{ Winter 2016} \\
\hline & $\begin{array}{l}\text { CWU } \\
\left(\mathrm{m}^{3} \mathrm{ha}^{-1}\right)\end{array}$ & $\begin{array}{l}\text { Grain } \\
\text { Yield } \\
\left(\mathrm{kg} \mathrm{ha}^{-1}\right)\end{array}$ & $\begin{array}{l}\text { WUE } \\
(\mathbf{k g} \\
\left.\mathbf{m m}^{-1}\right)\end{array}$ & $\begin{array}{l}\mathbf{E W P} \\
\left(\mathbf{M K} / \mathrm{m}^{3}\right)\end{array}$ & $\begin{array}{l}\text { CWU } \\
\left(\mathrm{m}^{3} \mathrm{ha}^{-1}\right)\end{array}$ & $\begin{array}{l}\text { Grain } \\
\text { Yield } \\
\left(\mathrm{kg} \mathrm{ha}^{-1}\right)\end{array}$ & $\begin{array}{l}\text { WUE } \\
\left(\mathbf{k g}^{-1}\right) \\
\left.\mathbf{m m}^{-1}\right)\end{array}$ & $\begin{array}{l}\text { EWP } \\
\left(\mathrm{MK} / \mathrm{m}^{3}\right)\end{array}$ \\
\hline \multicolumn{9}{|c|}{ Every Furrow Irrigation } \\
\hline SC 719 & 6,515 & $9,666^{\mathrm{a}}$ & $14.8^{\mathrm{bc}}$ & 297 & 5,604 & 9,771 & 17.4 & 296.40 \\
\hline PAN4M19 & 6,251 & $7,450^{\mathrm{bc}}$ & 11.9 & 238 & 5,560 & 8,500 & 15.3 & 259.89 \\
\hline MH 31 & - & - & - & - & 5,459 & 8,531 & 15.6 & 265.67 \\
\hline MH 26 & 5,651 & $7,856^{\mathrm{b}}$ & $13.9^{\mathrm{cd}}$ & 278 & & & & \\
\hline DKC 90-89 & 5,706 & $6,827^{\mathrm{cd}}$ & $13.0^{\mathrm{d}}$ & 239 & 5,398 & 7,708 & 14.3 & 242.76 \\
\hline ZM 721 & 6,312 & $7,071^{\mathrm{bc}}$ & 11.2 & 224 & 5,555 & 9,073 & 16.3 & 277.66 \\
\hline PAN4M21 & - & - & - & - & 5,466 & 8,187 & 15.0 & 254.64 \\
\hline Mean & 6,087 & $\mathbf{7 , 7 7 4} 4^{\mathrm{A}}$ & $12.8^{\mathrm{B}}$ & 255 & 5,507 & 8,628 & 15.7 & 266.17 \\
\hline \multicolumn{9}{|c|}{ Alternate Furrow Irrigation } \\
\hline SC 719 & 4,426 & $7,003^{\text {cd }}$ & $15.9^{\mathrm{b}}$ & 316 & 3,859 & 9,615 & 24.9 & 423.55 \\
\hline PAN4M19 & 4,143 & $5,262^{\mathrm{f}}$ & $12.7^{\mathrm{d}}$ & 254 & 3,786 & 6,771 & 17.9 & 304.03 \\
\hline MH 31 & - & - & - & - & 3,688 & 7,073 & 19.2 & 326.03 \\
\hline MH 26 & 3,540 & $7,170^{\mathrm{b}}$ & $20.3^{\mathrm{a}}$ & 405 & & & & \\
\hline DKC 90-89 & 3,595 & $5,754^{\mathrm{ef}}$ & $16.0^{\mathrm{b}}$ & 320 & 3,599 & 6,427 & 17.9 & 303.59 \\
\hline ZM 721 & 4,217 & $6,132^{\mathrm{de}}$ & $14.5^{\mathrm{bcd}}$ & 291 & 3,800 & 6,521 & 17.2 & 292.72 \\
\hline PAN4M21 & - & - & - & - & 3,705 & 7,156 & 19.3 & 328.36 \\
\hline Mean & 4,024 & $6,264^{B}$ & $15.9^{A}$ & 317 & 3,739 & 7,260 & 19.4 & 329.54 \\
\hline Cv. (\%) & & 10.8 & 10.9 & 10.9 & & 15.0 & 14.4 & 14.4 \\
\hline \multicolumn{9}{|l|}{ Significance } \\
\hline Varieties & & $\mathrm{P}<0.001$ & $\mathrm{P}<0.001$ & $\mathrm{P}<0.001$ & & $\mathrm{P}<0.01$ & $\mathrm{P}<0.001$ & $\mathrm{P}<0.001$ \\
\hline Irrigation & & $\mathrm{P}<0.01$ & $\mathrm{P}<0.01$ & $\mathrm{P}<0.01$ & & $\mathrm{P}<0.01$ & $\mathrm{P}<0.01$ & $\mathrm{P}<0.01$ \\
\hline Interaction & & Ns & $\mathrm{P}<0.01$ & $\mathrm{P}<0.01$ & & - & - & - \\
\hline \multicolumn{9}{|l|}{$\mathbf{L S D}_{0.05}$} \\
\hline Varieties & & 784.91 & 1.618 & 32.4 & & $1,217.1$ & 2.573 & 43.762 \\
\hline Irrigation & & 468.85 & 1.398 & 28.0 & & 603.4 & 1.063 & 17.977 \\
\hline Interaction & & - & 2.228 & 26.3 & & - & - & - \\
\hline
\end{tabular}

Note: Columns with similar letters are not significantly different.

Rows with similar capital letters are not significantly different.

\subsection{Maize Grain Yield and WUE for Selected Maize Varieties at On-farm}

There were significant differences in maize grain yield between the irrigation strategies $(\mathrm{P}<0.05)$ and varieties $(\mathrm{P}<0.001)$ at Nanzolo irrigation scheme in 2015 (Table 6). Alternate furrow irrigated maize had lower yields compared to every furrow irrigated maize. SC719 had highest yields but there were not significantly different between AFI and EFI strategies. Maize grain yields did not differ between irrigation strategies and varieties at Bwanje, Chitsukwa irrigation scheme in 2015 (P>0.05). At Mpitilira irrigation scheme, differences were only observed between maize varieties but not between AFI and EFI. SC719 had the highest grain yield at EFI but not significantly different to yields at AFI (P>0.05). PHB30G19-6 followed by SC627 had high yield and WUE with the least in SC403 in 2014. SC719 and DKC9053 had high grain yield at On-Farm under AFI. Most varieties with high yield had less per cent reduction. Some late maize maturing varieties were much better under AFI despite long duration (Table 6). 
Table 6a. Response of Maize Varieties to AFI \& EFI at On - Farm Trials - Nanzolo Irrigation Scheme, 2015

\begin{tabular}{|c|c|c|c|c|c|}
\hline \multirow{2}{*}{$\begin{array}{l}\text { Irrigation Strategy } \\
\text { Maize Varieties }\end{array}$} & \multicolumn{2}{|c|}{ Every furrow irrigation } & \multicolumn{2}{|c|}{ Alternate Furrow Irrigation } & \multirow{2}{*}{$\begin{array}{l}\text { Percentage of } \\
\text { yield reduction } \\
\text { with AFI }\end{array}$} \\
\hline & $\begin{array}{l}\text { Amount of irrigation } \\
(\mathrm{mm})\end{array}$ & $\begin{array}{l}\text { Grain yield } \\
\left(\mathrm{kg} \mathrm{ha}^{-1}\right)\end{array}$ & $\begin{array}{l}\text { Amount of irrigation } \\
(\mathrm{mm})\end{array}$ & $\begin{array}{l}\text { Grain yield } \\
\left(\mathrm{kg} \mathrm{ha}^{-1}\right)\end{array}$ & \\
\hline DKC 8033 & 435.5 & $4,376^{\mathrm{c}}$ & 263.2 & $4,278^{\mathrm{b}}$ & -4.1 \\
\hline DKC 8053 & 435.5 & $4,536^{\mathrm{c}}$ & 263.2 & $3,785^{\mathrm{c}}$ & 8.3 \\
\hline DKC 9053 & 435.5 & $5,158^{\mathrm{b}}$ & 263.2 & $4,852^{\mathrm{a}}$ & 15.6 \\
\hline DKC 9089 & 435.5 & $4,381^{\mathrm{c}}$ & 263.2 & $3,925^{\mathrm{c}}$ & -1.0 \\
\hline SC 403 & 435.5 & $4,951^{\mathrm{b}}$ & 263.2 & $4,300^{\mathrm{b}}$ & 15.4 \\
\hline SC 537 & 435.5 & $4,714^{\mathrm{b}}$ & 263.2 & $4,266^{\mathrm{b}}$ & 11.9 \\
\hline SC719 & 435.5 & $6,338^{\mathrm{a}}$ & 263.2 & $5,122^{\mathrm{a}}$ & 27.6 \\
\hline Cv.(\%) & & & & 8.0 & \\
\hline \multirow[t]{2}{*}{ Sign. } & Irrigation & & & $\mathrm{P}<0.05$ & \\
\hline & Varieties & & & $\mathrm{P}<0.001$ & \\
\hline $\mathrm{LSD}_{0.05}$ & Irrigation & & & 533.5 & \\
\hline
\end{tabular}

Table 6b. Response of Maize varieties to AFI \& EFI at Chitsukwa Irrigation Scheme, Winter 2015

\begin{tabular}{|c|c|c|c|c|c|}
\hline \multirow{2}{*}{$\begin{array}{l}\text { Irrigation Strategy } \\
\text { Maize Varieties }\end{array}$} & \multicolumn{2}{|c|}{ Every furrow irrigation } & \multicolumn{2}{|c|}{ Alternate Furrow Irrigation } & \multirow{2}{*}{$\begin{array}{l}\text { Percentage of } \\
\text { yield reduction } \\
\text { with AFI }\end{array}$} \\
\hline & $\begin{array}{l}\text { Amount of irrigation } \\
(\mathrm{mm})\end{array}$ & $\begin{array}{l}\text { Grain yield } \\
\left(\mathrm{kg} \mathrm{ha}^{-1}\right)\end{array}$ & $\begin{array}{l}\text { Amount of irrigation } \\
(\mathrm{mm})\end{array}$ & $\begin{array}{l}\text { Grain yield } \\
\left(\mathrm{kg} \mathrm{ha}^{-1}\right)\end{array}$ & \\
\hline DKC 8033 & 496 & 6,001 & 280 & 6,503 & -7.98 \\
\hline DKC 9053 & 496 & 5,890 & 280 & 6,088 & -9.89 \\
\hline DKC 9089 & 496 & 5,500 & 280 & 5,646 & -9.20 \\
\hline SC 403 & 496 & 5,518 & 280 & 7,076 & -52.85 \\
\hline SC 537 & 496 & 4,550 & 280 & 6,430 & -58.99 \\
\hline SC719 & 496 & 5,250 & 280 & 6,719 & -43.02 \\
\hline Cv. (\%) & & & & 15.9 & \\
\hline Irrigation & & & & Ns & \\
\hline Maize varieties & & & & Ns & \\
\hline
\end{tabular}

Table 6c. Response of Maize varieties to EFI and AFI at Bwanje Irrigation Scheme, Winter 2015

\begin{tabular}{|c|c|c|c|c|c|}
\hline \multirow{2}{*}{$\begin{array}{l}\text { Irrigation Strategy } \\
\text { Maize Varieties }\end{array}$} & \multicolumn{2}{|c|}{ Every furrow irrigation } & \multicolumn{2}{|c|}{ Alternate Furrow Irrigation } & \multirow{2}{*}{$\begin{array}{l}\text { Percentage of } \\
\text { yield reduction } \\
\text { with AFI }\end{array}$} \\
\hline & $\begin{array}{l}\text { Amount of irrigation } \\
(\mathrm{mm})\end{array}$ & $\begin{array}{l}\text { Grain yield } \\
\left(\mathrm{kg} \mathrm{ha}^{-1}\right)\end{array}$ & $\begin{array}{l}\text { Amount of irrigation } \\
(\mathrm{mm})\end{array}$ & $\begin{array}{l}\text { Grain yield } \\
\left(\mathrm{kg} \mathrm{ha}^{-1}\right)\end{array}$ & \\
\hline DKC8033 & & 5,955 & & 4,714 & $20 . \%$ \\
\hline DKC8053 & & 4,518 & & 6,074 & $-34.4 \%$ \\
\hline DKC9053 & & 6,194 & & 6,608 & $-6.7 \%$ \\
\hline DKC9089 & & 5,360 & & 5,732 & $-6.9 \%$ \\
\hline SC403 & & 6,064 & & 5,615 & $\mathbf{7 . 4 \%}$ \\
\hline SC537 & & 6,008 & & 5,908 & $1.7 \%$ \\
\hline SC719 & & 7,885 & & 5,560 & $29.5 \%$ \\
\hline $\mathrm{CV}(\%)$ & & & & 23.5 & \\
\hline Sign. & Irrigation & & & Ns & \\
\hline & Maize varieties & & & Ns & \\
\hline
\end{tabular}


Table 6d. Response of maize varieties to EFI and AFI at Mpitilira Irrigation Scheme, Winter 2015

\begin{tabular}{llllll}
\hline Irrigation Strategy & \multicolumn{2}{c}{ Every furrow irrigation } & \multicolumn{2}{c}{ Alternate Furrow Irrigation } & Percentage of \\
Maize Varieties & $\begin{array}{l}\text { Amount of irrigation } \\
(\mathrm{mm})\end{array}$ & $\begin{array}{l}\text { Grain yield } \\
\left(\mathrm{kg} \mathrm{ha}^{-1}\right)\end{array}$ & $\begin{array}{l}\text { Amount of irrigation } \\
(\mathrm{mm})\end{array}$ & $\begin{array}{l}\text { Grain yield } \\
\left(\mathrm{kg} \mathrm{ha}^{-1}\right)\end{array}$ & $\begin{array}{l}\text { yield reduction } \\
\text { with AFI }\end{array}$ \\
\hline DKC8033 & & $5,169^{\mathrm{b}}$ & & $5,276^{\mathrm{b}}$ & -2.07 \\
DKC8053 & & $5,016^{\mathrm{b}}$ & & $5,291^{\mathrm{b}}$ & -5.48 \\
DKC9053 & & $5,123^{\mathrm{b}}$ & & $5,519^{\mathrm{a}}$ & -7.73 \\
DKC9089 & & $5,051^{\mathrm{b}}$ & & $5,061^{\mathrm{b}}$ & -0.20 \\
SC403 & & $5,309^{\mathrm{b}}$ & & $5,650^{\mathrm{a}}$ & -6.42 \\
SC537 & & $5,286^{\mathrm{b}}$ & $5,775^{\mathrm{a}}$ & -9.25 \\
SC719 & & $6,422^{\mathrm{a}}$ & & $5,978^{\mathrm{a}}$ & 6.91 \\
Cv. $(\%)$ & Irrigation & & & 12.5 & \\
Sign. & Maize varieties & & & $\mathrm{Ns}$ & \\
& Maize varieties & & & $\mathrm{P}<0.01$ & \\
LSD $_{0.05}$ & & & 554.7 & \\
\hline
\end{tabular}

\section{Conclusion and Recommendation}

This study acknowledges that application of alternate furrow irrigation strategy save water without affecting maize grain yield. Only late maturing maize varieties proved to have minor yield reduction with AFI in 2015 and 2016. These results were in line with our null hypothesis 'AFI strategy improves WUE without greatly reducing maize grain yield.' This proves that the conventional way of furrow irrigation, EFI used by small scale farmers uses water luxuriously and that does not increase yield but increases water pumping costs and environmental degradation. On the other hand, late maturing results (2015 and 2016) partially challenged our hypothesis by indicating that water needs of maize may not be met by AFI because it imposes greater water stress that decreases maize grain yields. A larger part of the study concluded that AFI is a promising furrow irrigation water management strategy for reducing water use in irrigation schemes in Malawi. The one-year contradiction has been clarified by categorising maize varieties according to their maturing period.

For these reasons AFI technique was recommended to smallholder farmers on the following condition - that AFI is applied fully on early and medium maturing varieties within 7 - days irrigation interval. For late maturing maize varieties, alternate furrow irrigation technique should be applied from initial stage to mid - stage (up 55 days from planting) then apply every furrow irrigation at tasseling and silking stages to reduce water stress at this critical stage.

\section{Acknowledgements}

I wish to express my heartfelt indebtedness to the technical team comprising of Mr. Raphael Banda, Mr. Horace Khakiwa, Charles Nthewa, Mr. Helbert Kumwenda and Jonathan Chikankheni who gave enthusiastic and motivating technical support throughout this project. I also thank Department of Agricultural Research Services, Department of Irrigation and Department of Agricultural Engineering at LUANAR for mutual and solitary efforts. I also thank Dr Hendrix Kazembe who gave major contribution and momentum to the project as Soils and Agricultural Engineering National Research Coordinator that time. Many thanks also go to Irrigation \& Drainage Research Attendants and staff of Kasinthula Agricultural Research Station with whom we shared knowledge and work in many different ways to accomplish the project objectives.

Finally, I would also like to acknowledge Agricultural Productivity Program for Southern Africa (APPSA) for funding and The Centre for Agricultural Research and Development for Southern Africa (CCARDESA) for administration of APPSA R \& D Project entitled "Improving water use efficiency in maize production" that has helped to study Alternate Furrow Irrigation technique at on - farm level at five different irrigation schemes in Malawi.

\section{References}

Allen, R. G., Pereira, L. S., Raes, D., \& Smith, M. (1998). Crop evapotranspiration. Guidelines for computing crop water requirements. FAO Irrigation and Drainage Paper 56.Rome, Italy: Food and Agriculture Organization of the United Nations (FAO).

Ali Reza Sepaskhah \& Mohammad Hasan Khajehabdollahi (2005). Alternate Furrow Irrigation with Different Irrigation Intervals for Maize (Zea mays L.), Plant Production Science, 8(5), 592-600.

https://doi.org/10.1626/pps.8.592 
Barker, R., Dawe, D., \& Inocencio, A. (2003). Economics of water productivity in managing water for agriculture In W. Kijne, R. Barker, \& D. Molden (Eds.), Water productivity in Agriculture: Limits and opportunities for improvement (pp. 19-37). CAB International, Wallingford, UK. https://doi.org/10.1079/9780851996691.0019

Dass, A., Chandra, S., Choudhary, A. K., Singh, G., \& Sudhishri, S. (2016). Influence of field re-ponding pattern and plant spacing on rice root-shoot characteristics, yield, and water productivity of two modern cultivars under SRI management in Indian Mollisols. Paddy and Water Environment, 14, 45-59. https://doi.org/10.1007/s10333-015-0477-z

Fandika, I. R., Kadyampakeni, D. M., Bottomani, C., \& Kakhiwa, H. (2007). Comparative response of irrigated maize to organic and inorganic fertilizer application. Physics and Chemistry of the Earth, 32(15-18), 1107-1116. https://doi.org/10.1016/j.pce.2007.07.025

Gurvinder, S.,, Joshi, V. K., Chandra, S., Bhatnagar, A., \& Dass, A. (2016). Spring maize (Zea mays L.) response to different crop establishment and moisture management practices in north-west plains of India. Research on Crops, 17(2), 226-230. https://doi.org/10.5958/2348-7542.2016.00039.5

Hoekstra, A. Y., Chapagain, A. K., Aldaya, M. M., \& Mekonnen, M. M. (2009). Water footprint manual: State of the art 2009. Enschede, The Netherlands.

Jakhar, P., Rana, K. S., Dass, A., Choudhary, A. K., \& Choudhary, M. (2017). Influence of moisture management practices on productivity, profitability and energy dynamics of rainfed maize (Zea mays L.) in semi-arid sub-tropical climate. Indian Journal of Agronomy, 62(2), 191-196.

Jones \& Cowan, I. R. (1982). Regulation of water use in relation to carbon gain on higher plants. In O. L. Lange, et al. (Eds.), Physiological Plant Ecology II (pp. 589-614). Springer, Berlin, Cowan. https://doi.org/10.1007/978-3-642-68150-9_18

Jones, H. G. (1992). Plants and microclimate: a quantitative approach to environmental plant physiology, 2nd edn. Cambridge: Cambridge University Press.

Kadyampakeni, D. M. S., \& Fandika, I. R. (2008). A review of irrigation and drainage in Malawi. In Land and Water Management in Southern Africa: Towards sustainable Agriculture. Edited by Patrick Rankhumise, Calvin Nhira and Alfred Mapiki. Pretoria, South Africa.

Kayikcioglu, Husseyn Husnu (2012) Short-term effects of irrigation with treated domestic wastewater on microbiological activity of a Vertic xerofluvent soil under Mediterranean conditions. Journal of environmental management.102:108 - 114

Kang, S., Liang, Z., Hu, W., \& Zhang, J. (1998). Water use efficiency of controlled root-division alternate irrigation on maize plants. Agricultural Water Management, 38(1998), 69-76. https://doi.org/10.1016/S0378-3774(98)00048-1

Kang, S., Liang, Z., Pan, Y., Shi, P., \& Zhang, J. (1999). Alternate furrow irrigation for maize production in an arid area. Agricultural Water Management, 45(3), 267-274. https://doi.org/10.1016/S0378-3774(00)00072-X

Kumari, K., Dass, A., Sudhishri, S., Kaur, R., \& Rani, A. (2017). Effect of irrigation regimes and variable nitrogen rates on yield components and nutrient uptake in maize (Zea mays L.) in north-Indian plains. Indian Journal of Agronomy, 62(1), 104-107.

Kumari, K., Dass, A., Sudhishri, S., Meena, M. C., Jinger, D., Kumar, A., \& Kumar, S. (2016). Root-shoot characteristics, yield and soil nitrogen status under different irrigation and nitrogen levels in maize (Zea mays L.). Annals of Agricultural Research, 37(4), 383-390.

Liu, C, Zhang, X., \& Zhang, Y. (2002). Determination of daily evaporation and evapotranspiration of winter wheat and maize by large-scale weighed lysimeter and micro-lysimeter. Agricultural and Forest Meteorology, 111(2), 109-120. https://doi.org/10.1016/S0168-1923(02)00015-1

Meier, U. (2006). A note on the power of Fisher's least significant difference procedure. Pharmaceutical Statistics, 5(4), 253-263. https://doi.org/10.1002/pst.210

Mitchell, A. R., Light, J. E., \& Tera, P. (1992). Alternate and alternating furrow irrigation of peppermint to minimize nitrate leaching. Central Oregon Agricultural Research Center Madras, OR.

Premrov, A., Schulte, R. P. O., Coxon, C. E., Hackett, R., \& Richards, K. G. (2010). Predicting soil moisture conditions for arable free draining soils in Ireland under spring cereal crop production.Irish Journal of 
Agricultural and Food Research, 49(2), 99-113.

Rafiee, M. (2012). Effect of every other furrow irrigation and planting density on physiological traits in corn (Zea mays). World applied science Journal, 17(2), 189-193.

Rana, D. S., Dass, A., \& Rajanna, G. A. (2016). Biotic and abiotic stress management in pulses. Indian Journal of Agronomy, 61: 4th IAC Special Issue, 238-247.

SAS. (2008). SAS Procedures Guide - Version 9.2 Edition.

Sepaskhah, A. R., \& Ghasemi, M. M. (2008). Every - Other - Furrow irrigation with different irrigation intervals for grain sorghum. Pakistan journal of Biological Sciences, 11, 1234-1239. https://doi.org/10.3923/pjbs.2008.1234.1239

Stewart, M., Hodges, G., Stone, J. F., \& Nofziger, D. L. (1993). Water use and yields of cotton grown under wide-spaced furrow irrigation. Agric. Wat. Manage, 24, 27-38.

https://doi.org/10.1016/0378-3774(93)90059-J

\section{Copyrights}

Copyright for this article is retained by the author(s), with first publication rights granted to the journal.

This is an open-access article distributed under the terms and conditions of the Creative Commons Attribution license (http://creativecommons.org/licenses/by/3.0/). 